\title{
Trends in the Control Schemes for Bilateral Teleoperation with Time Delay
}

\author{
Jiayi Zhu ${ }^{1}$, Xiaochuan $\mathrm{He}^{2}$, and Wail Gueaieb ${ }^{1}$ \\ 1 Machine Intelligence, Robotics and Mechatronics (MIRaM) Laboratory \\ School of Information Technology and Engineering (SITE) \\ University of Ottawa, Ottawa ON K1N 6N5, Canada \\ 2 Studies on Teleoperation and Advanced Robotics (STAR) Laboratory \\ Department of Systems and Computer Engineering \\ Carleton University, Ottawa ON K1S 5B6, Canada
}

\begin{abstract}
In the last two decades, an increasing interest has been observed in the development of effective bilateral teleoperation systems. One of the biggest challenges encountered however is the presence of time delay, which can cause instability and poor transparency to the system. Researchers have been trying to tackle this problem from different angles and various control schemes have been proposed and tested. This paper aims to do an overview of those schemes and to compare them based on their assessment in order to give new researchers a summary of the current state of art. The schemes are divided into four main categories: passivity based, prediction based, sliding-mode control schemes and others. The comparison is based on transparency level, stability requirement, delay type, and delay range.
\end{abstract}

Keywords: bilateral teleoperation, time delay, delayed teleoperation, control scheme, passivity.

\section{Introduction}

Teleoperation is an action performed over distance where the human operator at the local site can control a robot at the remote site. When such an operation is qualified as bilateral, the operator can perceive the corresponding force feedback when the remote robot enters in contact with its environment. In the literature, the robot directly manipulated by the human operator is called "master" and the one located at the remote site is called "slave". A basic block diagram of such a system is shown in figure 1.

Such a system has a large potential with possible applications in nuclear, mining, space, medicine and many other fields. Unilateral teleoperation where the force feedback is non-existent is already in service in those fields; however as mentioned in [20], if force feedback was incorporated in those systems, it could ameliorate the completion time and quality of the overall teleoperated task. The incorporation of force feedback in the control system is unfortunately challenging, especially in the presence of a time delay which may cause inefficiency to the human operator and instability to the system. 


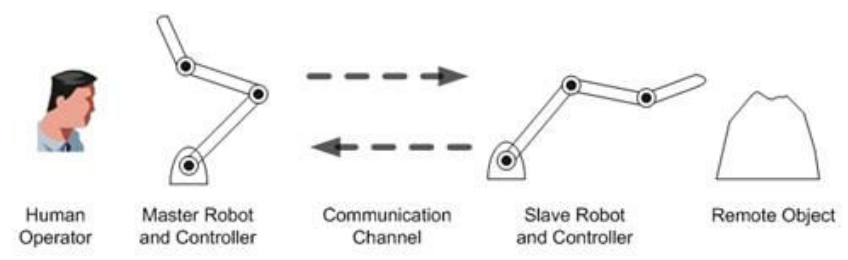

Fig. 1. An illustration of the bilateral teleoperation system

A few papers have done surveys of teleoperation control schemes in $[2,4,8,11$, $15,16,19]$; however none was found to be specifically oriented towards both bilateral and time-delayed control schemes. Also, a sorting of the control schemes into categories is desirable since a growing number of controllers is being suggested. Furthermore, this work aims to provide a more conceptual explanation of the control schemes in order to ensure the provision of a comprehensive introduction suitable for new researchers in this field.

In this paper, a variety of proposed control schemes will be examined with the aim of introducing readers to the different control methods. Each concept will be presented and their pros and cons investigated.

This work will be divided into different parts, section 2 will give a background of time delay in teleoperation system, section 3 will talk about the different control schemes that have been proposed in the literature along with their features, section 4 gives a few remarks about the trends of the future works in this field, and section 5 will end with a conclusion.

\section{Time Delay in Teleoperation}

Time delay in teleoperation systems can be caused by the potentially large physical distance between the master and slave robots, the processing time of the controllers, the network congestion, and the communication bandwidth. It has been shown as early as 1966 in [5] that even a delay less than $50 \mathrm{~ms}$ can destabilize a bilateral teleoperation system. It must be mentioned however that time delay is not the only cause of instability of a teleoperation system. In fact, other sources include noise, external disturbances, force scaling, non passive environment, etc. But this paper will specifically focus on the problems caused by time-delay and the suggested solutions.

Beside instability, time delay may cause the human operator to be unable to instantaneously perceive her/his action and its corresponding reaction. This would decay the quality of a teleoperated task. One of the first methods that appeared to deal with time delayed teleoperation system uses the "move and wait" strategy where open-loop control is used as mentioned in [18]. The system is guaranteed to be stable in this case, however this method is very time consuming and is tiring for the operator.

In the literature, there are usually two categories of time delays considered: constant and time-varying. The constant time delay is usually in the order of 
less than one second, and it can find its application in master-slave robots that are physically linked, either by electrical wires or by optical fibers. On the other hand, the variable time delay is relevant to the applications using Internet as a communication channel. Due to the constantly changing condition of the network, time delay can vary between $0.1 \mathrm{~s}$ to $3 \mathrm{~s}[20]$.

\section{Control Schemes for Time-Delayed Bilateral Teleoperation}

In the literature, various control schemes are proposed for bilateral operation. All those presented in this paper appeared in the last two decades and have been experimentally verified. The comparison will be done for delay type and range, and also for the two most critical design criteria of a teleoperation control scheme: transparency and stability. Transparency refers to the accuracy in the position and force tracking. Ideally, a perfect transparency would make the human operator feel and believe that he or she is at the remote site. Stability on the other hand refers to the fact that the system output would always be bounded for a bounded input. In the close-loop control structures, this means that the poles should all be on the left hand side of the s-plane. Those two criteria often need to be traded off since a high bandwidth allow high transparency at the cost of degrading stability due to the time delay.

\subsection{Passivity-Based Schemes}

In order to ensure the overall system stability, its passivity can be used as the design objective. Passivity is a sufficient condition for stability. A passive system is one that can only consume energy without being able to produce any. In a two-port telerobotic system where the energy flow is characterized by $\dot{x}(t)=\left[\dot{x}_{1}(t), \dot{x}_{2}(t)\right]^{T}$ and effort by $F(t)=\left[F_{1}(t), F_{2}(t)\right]^{T}$ ( $\dot{x}$ represents velocity, $F$ represents force, 1 represents the master side, and 2 represents the slave side), this implies:

$$
\int_{0}^{\infty} F^{T}(t) \dot{x}(t) d t \geq 0 .
$$

The passivity-based control was first proposed by Anderson and Spong [1] and was considered a breakthrough by many researchers. In this method, the communication channel of the system was redefined in order to guarantee passivity independent of time delay. This was done by combining network theory, scattering transformation and passivity. Two years later, Niemeyer and Slotine further built into the idea by introducing wave variables [12]. The wave transformation converges the power variables (force and velocity) into wave variables as follow:

$$
u=\frac{b \dot{x}+F}{\sqrt{2 b}}, v=\frac{b \dot{x}-F}{\sqrt{2 b}},
$$

where $u$ and $v$ are the wave variables, while $\dot{x}$ and $F$ are the power variables velocity and force, respectively. The parameter $b$ is the wave impedance and can 
be tuned to modify the system's behaviour. Stability problem is solved under the assumption that each blocks of the overall system is passive, including human operator, environment, controllers, robots and communication channel.

This control scheme was experimented using the hard contact and back drivability tests. The results show that the constant time delay can be in the range of $40 \mathrm{~ms}$ to $2 \mathrm{~s}$ without destabilizing the system. But the tracking performance of the position and force commands (transparency) of the system was quite poor. When the time delay was increased to $2 \mathrm{~s}$, the force and position tracking began to severely degrade.

Those problems may be caused by wave reflection, discrete sampling, numerical errors, and data loss. To solve them, Niemeyer and Slotine proposed two possible solutions in 2004 [13]: the first was to use wave integral, where no extra bandwidth was required, but the system can transmit position, velocity and force information (instead of just velocity and force); the second solution was the addition of a corrective term in response to the drift. It introduced no extra power, and slowly compensated for the position errors. The wave integral method has a potential to destabilize system under time-varying delays, therefore it was also proposed to include a reconstruction filter in the design. Another problem addressed by Niemeyer and Slotine was wave reflection, which was the issue of wave variables circulating several time in the system before dying out, compromising system's transparency. They suggested to use impedance matching where each subsystem is designed as a damper before the impedance is chosen and adjusted. This control scheme was tested using a constant time delay of up to $2 \mathrm{~s}$ and then using a variable delay from $50 \mathrm{~ms}$ to $1 \mathrm{~s}$. A contact between slave robot and remote environment was included in the test. The system is shown to be stable and the transparency is improved compared to the basic wave variable scheme. However the position error between master and slave robots was observed to be in the order of centimetres.

In 2006, Lee and Spong proposed a new control framework in [10], which utilized the simple proportional-derivative (PD) control for bilateral teleoperation of a pair of multi-degree-of-freedom nonlinear robotic systems. In contrast to the scattering theory, this framework passifies the combination of the communication and control blocks altogether. It can exploit the benefits of position feedback while keeping the system passive. This method was also used for constant time delay, and the parameters of the PD control system were required to be optimized.

The control scheme developed by Lee and Spong used passivity concept, the Lyapunov-Krasovskii technique, and Parsevals identity with any finite constant time delays. They introduced several control parameters in order to achieve the master-slave coordination, force reflection in both ways, and the passivity of the system. For the experiments, four scenarios were assumed. When the time delay was $3 \mathrm{~s}$, the human operator could perceive the force reflection from the object. So the stability and transparency of the teleoperation system could be guaranteed. However, it can be noticed in the experiments for this control scheme 
that some errors occurred in both the force reflection and position coordination, which were due to the friction used in the algorithm.

A combination of wave variables and predictors, along with the addition of drift control was proposed in 2006 [3]. It claims stability due to the use of wavevariable transfer over the transmission channel and also due to the assumption stating that slave robot dissipates enough energy. It switches between two different predictors depending on the type of remote environment encountered. The semi-adaptive predictor is superior in rigid contact environment while the fulladaptive predictor is better suited for slow varying environment. As shown by the experiments, the transparency is much better with this combined control method than the individual ones.

The varying time delay used in the experiment was between $0.4 \mathrm{~s}$ to $0.5 \mathrm{~s}$. Additionally, the researchers were modelling UDP transmission protocol with $10 \%$ chance of data loss and system showed to be robust towards this transmission model. In general, the tracking performance was quite satisfactory even though not extremely precise, with the exception of the position tracking during freespace which proves to be quite accurate despite small oscillations observed in the slave robot. One of its weaknesses is its assumptions for guaranteeing passivity under time-varying delays. It is presumed that the slave robot is capable of dissipating enough energy. In reality, this may or may not be a good assumption. This is one of the few methods tested under realistic UDP environment and the system showed robustness towards this transmission model.

The biggest advantage of the wave variable based methods is that they guarantee stability and are completely robust to delays. Also, the wave commands control the output energy in terms of strength and direction only, so it can converge to either motion or force. Hence, the controller can be the same for when manipulator is in free space and when it is in contact with the environment. Additionally, those methods don't require any knowledge of the system since stability is evaluated based on input and output only. The main disadvantage of this control method is the assumption of human operator and environment being both passive, which may not be always be true.

Passivity is a sufficient condition for system stability. Therefore the passivity based control schemes reviewed in this paper could all guarantee the stability of the bilateral teleoperation system. When the system is tested under constant time delay, the scattering theory plays an important role in the scheme design. While when the delay of the system is varying, some extra variables, control parameters, or special communication channels are needed to improve the stability and the tracking performance of the system.

\subsection{Prediction Based Schemes}

Just around 2000, the concept of Smith Controller, which was first proposed to deal with delays in chemical plants in 1957, has been applied to bilateral teleoperation systems. In this method, the main idea is to replicate the response of the slave and the environment on the master side by building a local model. Hence this predictive local model is a representation of the remote object. Once 
established, the model permits to completely replicate the response of the remote object, taking out the negative effects of time delay. This kind of control can be robust against time delay of above $1 \mathrm{~s}$, which is a greatly desired feature especially for space teleoperation. However it requires a very good model of the environment in order to reach good transparency. Along with the stability of the overall system, those are the two main challenges that researchers have been trying to overcome. The authors of this paper think that the presence of the model makes the relationship between transparency and stability to be no longer a clear trade-off. Because assuming that the model is a perfect representation of the remote site, the presence of the model guarantees the transparency of the system. Therefore stability remains as the only concern.

In 2003, Huang and Lewis proposed a control scheme based on this concept. In [9], they presented a two-channel position-position control architecture and used a neural network estimator on the slave side to estimate its dynamics. The neural network estimator is an adaptive system that is used to model complex relationships between inputs and outputs. It is widely employed in identification and control of nonlinear systems. This estimator builds up the model on the slave side by linearizing the nonlinear dynamics using local nonlinear compensation method and then sends this information back to the master side. The master side consists of a linear Smith predictor and it takes the information sent from the model in the slave side. At this point, all the nonlinear dynamics of the slave have been approximated to linear by the model. Since the model is transferring the information to the Smith predictor, the predictor is considered to give an accurate representation of the remote environment. Under the assumption that the slave dynamics is well modelled, this control scheme gives satisfactory results. However this can be justified in case of slowly varying or unchanging dynamics only since the predictor model is fixed; this is an important limitation of the system in some applications.

For this control scheme, the stability of the closed-loop system was proven using Lyapunov's method. A few important assumptions to ensure stability include the bounds on the desired states and on the output of the system. Also it assumes that the remote site is well represented by its linearized model. If this was not the case, system may be destabilized. This kind of control is suitable for systems with constant time-delays and gives satisfactory performance for freemotion tracking. However it suffers from the problem of poor transparency at contact, which is a common drawback for position-position control schemes. The results based on this kind of control scheme are obtained using a constant delay of $0.5 \mathrm{~s}$.

Around the same time, Fite, Goldfarb and Rubio proposed a predictive control scheme using force-position architecture [6]. They suggested the implementation of an adaptive controller at the master side in order to adjust the linear estimate on the master-side itself. In this case, the slave information fed back through the communication channel and is then constantly updating the model (predictor) on the master side. This gives the control scheme an asset of being adjustable to time varying environment. However, once again the predictor used 
here is still linear and it is important to note that not all the environment can be modeled perfectly with a linear model.

This scheme was not rigorously proven stable. It relies on the original idea of Smith predictor stating that if the model is a good representation of the remote site, system is stable. Because of the fact that an adaptive controller is used, this assumption is considered as good. Through the experiments, the transparency of the system is shown to be robust to changing environment dynamics for stiffness ranging from $75 \mathrm{~N} \cdot \mathrm{m}^{-1}$ to $1275 \mathrm{~N} \cdot \mathrm{m}^{-1}$. The type of delay discussed by the researchers is assumed to be constant and the conducted experiments were using a value of $0.5 \mathrm{~s}$ for each one-way time delay.

In 2006, Smith and Hashtrudi-Zaad presented an improved prediction-based model in [20] using neural network estimators on both master and slave sides, making both of them adaptive. It still uses force-position architecture as the one mentioned in previous paragraph. It builds the model on-line on the slave side using delayed information from the master as input and the corresponding force response from the slave as output, thus making the model free from time delay effects. This model is then transmitted back to the master side and continuously updated. This control scheme permits a real-time mapping of dynamic and nonlinear environment, but it needs a small learning period to build up the model.

In this case, the stability was also not analyzed theoretically. Also from the experiments conducted by the two researchers, even though the stability is shown to be much better compared to non-predictive methods, a noticeable position tracking error is still present. The constant time delay used in the experiments was $0.5 \mathrm{~s}$.

More recently in 2009, Yoshida and Namerikawa proposed another predictive control scheme in [22] and tried to solve the issues in the previous control schemes. Their idea is based on a 3 channel architecture where the delayed position and velocity information of the master are sent to the slave side, and the estimated environment parameters are sent back to the master for the predictor. It guarantees the stability of the system and also ensures that the position tracking error converges to zero while the force tracking error stays small.

For this type of control scheme discussed, the asymptotic stability was proven using Lyapunov method with the finding of a storage function. This is under the assumption that the parameter estimation errors and the master states are bounded. The experimental results show very small tracking errors once steady state is reached, however this system requires a potentially large convergence time in the beginning ( $12 \mathrm{~s}$ in the case presented) in order to ensure small to non-existent tracking error. The delay used by the authors was constant and had the value of $1 \mathrm{~s}$.

It can be seen that the predictive control schemes' main asset is being able to accommodate relatively large time delay. Their weakness however remains in the way of finding a good model of the remote site. The use of adaptive controllers and estimators seem to help modeling a non-linear and changing environment and show a much better outcome than the simple linearization method. 


\subsection{Sliding-Mode Control Schemes}

Very different from the passivity based control and the prediction based control, the sliding mode control is a nonlinear system control method which determines a sliding surface and forces the trajectories of the dynamic system to approach it, reach it, and stay on it. Several researchers have tried to use sliding mode based methods in order to ameliorate the bilateral teleoperation system.

In 1999, a modified sliding-mode controller for the bilateral teleoperation was suggested by Park and Cho [14]. In the literature, the sliding-mode controller is known to be robust to model parameter variations, hence has great applications for systems dealing with time-varying delay. In this case, position-force architecture is used. An impedance controller was implemented on the master side while two types of controllers were used on the slave side. In free space, a modified first order sliding-mode controller is employed and upon contact, a local compliance controller is applied. The nonlinear control gain of the modified sliding-mode controller was modified to be independent of the time delay, which ameliorated the transparency and stability of the system.

For this scheme, stability was not mathematically proven. Transparency seems to be acceptable according to the experimental results. The control scheme deals with varying time delay, and the forward delay from master to slave is considered to be different from the feedback delay from slave to master. The round-trip varying time delay used by Park and Cho in their simulations had a maximum value of as high as 4 seconds, hence showing a lot of potential for teleopearations with varying time-delay. A TCP network protocol is used, therefore no data was lost and no packet ordering was necessary.

Along the same line, an observer-based sliding mode control was proposed a few years later where the impedance control is used for the master and a secondorder sliding-mode control for the slave [7]. Even though it is a second order sliding mode control, the value of acceleration is not measured with sensor but is obtained with an observer. Using the same principle, the sensor measurement of velocity is also not required.

The resulting system was developed for constant unknown time delay. In free space, the output position, velocity and acceleration tend to the desired ones, however upon contact there exists a trade-off between force and position tracking. Moreover, the system does not achieve a perfect transparency, it only gives a good idea about the remote environment.

\subsection{Other Control Schemes}

A few other control schemes for bilateral teleoperation also appeared in the literature including an adaptive controller by Zhu and Salcudean [23], eventbased controller [17], gain-scheduled compensation [21], etc. Those will not be looked at in details in this paper due to space limitation. 


\section{Remarks About Future Trends}

Time delayed bilateral teleoperation is a fast progressing field of research. Based on its current status, a few remarks are made about its future trends.

It can be seen that an emerging interest has been shown from constant time delay to variable time delay, which models the Internet as the communication channel. In fact, due to its high availability and low cost, it has a great potential and has been a research interest since the late $90 \mathrm{~s}$. However in order to implement it, further research where time delay is implemented along with information loss and packet ordering (in case of UDP) needs to be done.

The time delays that were examined in the literature are mainly either constant and small (for physically linked master and slave robots) or varying up to a few seconds (for Internet-based applications). However there is also another type of delay which occurs in the case of space application. When the human operator is on Earth manipulating a robot in the space, the time delay can be much longer, even up to the range minutes. This application would mainly be useful for exploration missions on the Moon or Mars. More tests will need to be done if bilateral teleoperation is to be implemented in such applications.

Also, time-delayed bilateral teleoperation involving multiple human operators (many to one control), multiple slave manipulators (one to many), or both (many to many) would be a very useful control situation to be studied. Cooperation between operators is definitely needed in many contexts.

Moreover, scaled bilateral teleoperation system represents another challenge. In the medical applications, it is sometimes desirable to scale down and perform teleoperation on a micro and even nano scale. In other applications, it may be necessary to scale up, for example to multiply the human operators applied force.

\section{Conclusion}

Bilateral teleoperation has potentials in many research and industrial areas. As of now, the problem of time delay is one of the difficulties preventing this application to be implemented. A number of control schemes specific to this kind of manipulation were presented in this paper. They were divided into different categories and their pros and cons discussed. The comparison for the control schemes was also done, based on transparency level, stability requirement, delay type, and delay range. They demonstrate the main trends in the field of bilateral teleoperation and prove its development and its maturing steps.

\section{References}

1. Anderson, R., Spong, M.: Bilateral control of teleoperators with time delay. IEEE Transactions on Automatic Control 34(5), 494-501 (1989)

2. Arcara, P., Melchiorri, C.: Control schemes for teleoperation with time delay: a comparative study. Robotics and Autonomous Systems 38(1), 49-64 (1 2002) 
3. Ching, H., Book, W.: Internet-based bilateral teleoperation based on wave variable with adaptive predictor and direct driff control. Transactions of the ASME, Journal of Dynamic Systems, Measurement and Control 128, 86-93 (2006)

4. Ferre, M., Buss, M., Aracil, R., Melchiorri, C., Balaguer, C.: Advances in Telerobotics. Springer-Verlag Berlin Herdelberg, Germany (2007)

5. Ferrell, W.: Delayed force feedback. Hum. Factors 8(5), 449-455 (1966)

6. Fite, K., Goldfarb, M., Rubio, A.: Transparent telemanipulation in the presence of time delay. Proceedings of the IEEE/ASME International Conference on Advanced Intelligent Mechatronics 1, 254-259 (2003)

7. Garcia-Valdovinos, L., Parra-Vega, V., Arteaga, M.: Observer-based higher-order sliding mode impedance control of bilateral teleoperation under constant unknown time delay. In: Intelligent Robots and Systems, 2006 IEEE/RSJ International Conference on. pp. 1692-1699 (2006)

8. Hokayem, P., Spong, M.: Bilateral teleoperation: An historical survey. Automatica 42, 2035-2057 (2006)

9. Huang, J., Lewis, F.: Neural-network predictive control for nonlinear dynamic systems with time-delay. IEEE Trans. on Neural Networks 14(2), 377-389 (2003)

10. Lee, D., Spong, M.: Passive bilateral teleoperation with constant time delay. Proceeding of the 2006 IEEE International Conference on Robotics and Automation 22(2), 2902-2907 (2006)

11. Mulder, M.: Stability in haptic teleoperation literature review (2006)

12. Niemeyer, G., Slotine, J.: Stable adaptive teleoperation. IEEE Journal of Oceanic Engineering 16(1), 152-162 (1991)

13. Niemeyer, G., Slotine, J.: Telemanipulation with time delays. International Journal of Robotics Research 23(9), 873-890 (2004)

14. Park, J., Cho, H.: Sliding-mode control of bilateral teleoperation systems with force-reflection on the internet. In: Proceedings of the IEEE/RSJ International Conference on Intelligent Robots and System. vol. 2, pp. 1187-1192 (2000)

15. Rodriguez-Seda, E.: Comparative experimental study of control schemes for bilateral teleoperation systems. Master's thesis, Univ. Illinois (2007)

16. Rodriguez-Seda, E., Lee, D., Spong, M.: Experimental comparison study of control architectures for bilateral teleoperators. IEEE Transactions on Robotics 25(6), 1304-1318 (2009)

17. Sano, A., Fujimoto, H., Tanaka, M.: Gain-scheduled compensation for time delay of bilateral teleoperation systems. Robotics and Automation, 1998. Proceedings. 1998 IEEE International Conference on 3, 1916-1923 (1998)

18. Sheridan, T.: Space teleoperation through time delay: review and prognosis. Robotics and Automation, IEEE Transactions on 9(5), 592-606 (1993)

19. Sheridan, T.: Teleoperation, telerobotics and telepresence: A progress report. Control Eng. Pract. 3(3), 205-214 (1995)

20. Smith, A., Hashtrudi-Zaad, K.: Smith predictor type control architectures for time delayed teleoperation. The International Journal of Robotics Research 25(8), 797818 (August 2006)

21. Xi, N., Tarn, T.: Stability analysis of non-time referenced internet-based telerobotic systems. Robotics and Autonomous Systems 32(2-3), 173-178 (2000)

22. Yoshida, K., T.Namerikawa: Stability and tracking properties in predictive control with adaptation for bilateral teleoperation. In: Proceedings of the American Control Conference. pp. 1323-1328 (2009)

23. Zhu, W., Salcudean, S.: Stability guaranteed teleoperation: an adaptive motion/force control approach. IEEE Transactions on Automatic Control 45(11), 1951-1969 (2000) 